\title{
Sensibilidad a Aspectos Sonoros de las Palabras y su Relación con el Aprendizaje de la Escritura
}

\section{Sensitivity to Sound Aspects of Words and Their Relationship with Learning to Write}

\author{
Silvanne Ribeiro y Ana Teberosky \\ Universidad de Barcelona-España
}

\begin{abstract}
Resumen. En este artículo se investiga la relación entre la sensibilidad a los aspectos sonoros del lenguaje y los procesos de adquisición de la escritura en los niños de edad preescolar (4-6 años). La finalidad es estudiar diversas tareas que impliquen extracción y segmentación sobre la palabra, relacionadas con el proceso de aprendizaje de lo escrito. Más específicamente, estas tareas se refieren a la rima (Tarea 1) y a la segmentación (Tarea 2) en un contexto de juegos de palabras propuesto a niños preescolares. Los resultados muestran la existencia de una relación directa entre el procedimiento de extracción y segmentación y el nivel de conceptualización de lo escrito. En otras palabras, a mayor nivel de escritura, mayor trabajo de extracción de rima y de segmentación silábica. Encontramos pocas respuestas con segmentación fonémica, ésta no resultó frecuente incluso en los niños alfabéticos porque la comprensión del sistema no derivó automáticamente en segmentaciones exhaustivas.

Palabras clave: segmentación de palabras, conocimiento metalingüístico, adquisición de la escritura, rimas, preescolares.
\end{abstract}

\begin{abstract}
This article is intended to investigate the question of sensitivity to language and its sound aspects, as related to writing acquisition processes in preschool children (4 to 6 years old). The aim is to study certain tasks that imply extracting and segmenting words and to relate this to the process of learning to write. Specifically, these tasks relate to rhyme (Task 1) and segmentation (Task 2) in the context of word games presented to preschool children. The results show that there is a connection between the extraction and segmentation process and the level of conceptualization of what is written. The better at extracting rhyme and segmenting syllables the children were, the higher their level of writing. Only a few responses with phonemic segmentation were found, even in literate children, since understanding of the system did not automatically give rise to exhaustive segmentations.

Key words: word segmentation, metalinguistic knowledge, acquisition of writing, rhymes, preschool.
\end{abstract}

\section{Introducción}

En las últimas décadas los estudios sobre la sensibilidad a los aspectos sonoros del lenguaje y la conciencia fonológica han despertado un gran inte-

La correspondencia sobre este artículo debe enviarse a la primera autora al E-mail: naribeiro3@yahoo.es rés en muchos investigadores. Eso se debe a la relación demostrada entre conocimiento fonológico y desarrollo de la lectura y escritura.

La conciencia fonológica se refiere a la habilidad para segmentar la palabra en sonidos individuales, es decir en fonemas, así como la habilidad para agregar, sustituir o contar los fonemas que componen las palabras. Según Morais (1991), esa conciencia es 
una forma de conocimiento metalingüístico y está implicada la capacidad de realizar operaciones mentales sobre los sonidos del habla. Para Defior (1994), la conciencia fonológica no aparece repentinamente, sino que es el resultado de un aprendizaje gradual, cuando los niños desplazan su atención desde los aspectos significativos del lenguaje hacia la conciencia de su estructura y de las unidades o segmentos que lo componen. Analizar el lenguaje en sus componentes sonoros mínimos, es decir en fonemas, es una ardua tarea que no es necesaria durante el desarrollo del lenguaje oral, pero que se volverá inevitable durante el aprendizaje de la escritura. Diversos estudios han constatado que el desarrollo fonológico es un proceso lento, en el que los niños van evolucionando en el análisis y en la percepción de las unidades fonológicas de su lengua.

Numerosas investigaciones demuestran que la conciencia fonológica es una habilidad metalingüística importante en el aprendizaje de la lectura y de la escritura en lengua inglesa (Morais, 1991; Bryant y Bradley, 1998; Goswami, 1999), en castellano (Defior y Tudela, 1994), en hebreo (Share, 1995) y en portugués (Cardoso-Martins, 1995; Rego y Bryant, 1993). Dicha habilidad parece tener una fuerte relación con el éxito en el aprendizaje escolar (Bryant y Goswami, 1987; Bryant y Bradley, 1998), con el rendimiento en lectura (Morais, 1991) y también con los avances en la conceptualización de la escritura (Vernon, 1998; Vernon y Ferreiro, 1999). Estudios en los que han participado niños con dificultades en la lectura y los denominados disléxicos corroboran la afirmación de la importancia de la conciencia fonológica en la adquisición de la lectura y de la escritura, puesto que hay datos que afirman que los niños disléxicos no logran solucionar tareas fonológicas (Snowling, 1980).

Una importante discusión sobre la relación entre conciencia fonológica y aprendizaje ha sido planteada en términos de condición para el aprendizaje (Bryant y Bradley, 1983 y el grupo de Oxford), en términos de consecuencia (Morais, 1991; Alegría, 1993 y el grupo de Bruselas) o de relación recíproca (Stanovich, 1986). La línea de la presente investiga- ción se encuadra precisamente en ésta última, que es la más aceptada en la actualidad, en la cual se afirma que existe una relación de facilitación mutua entre conciencia fonológica y aprendizaje. En esta dirección apuntan algunos estudios que han demostrado que los niños poseen una gran sensibilidad a los sonidos del habla antes de la adquisición de la escritura (Morreira y Pontecorvo, 1998; Bryant, Maclean, Bradley, Crossland, 1989). Pero tal habilidad y sensibilidad para identificar variaciones, sean morfémicas o sonoras, no va acompañada de una habilidad equivalente para analizar y segmentar las palabras en sus componentes.

En efecto, diversos estudios han mostrado que los niños tienen una gran dificultad a la hora de segmentar las palabras en fonemas, aunque no en sílabas (Tolchinsky, Teberosky y Matas, 1993; Jiménez, 1992; Vernon, 1998). Según Carrillo y Serrano (1992, p. 52), eso se explica debido a que la unidad silábica posee propiedades acústicas que la hacen fácilmente distinguible tanto en la representación espectográfica del habla, como a nivel de percepción auditiva. De ahí que centrar la atención sobre esta unidad resulta mucho más fácil. En cambio, la unidad fonema resulta difícil para ellos porque los sonidos del lenguaje, a nivel de fonemas, resultan abstractos. Por otra parte, la dificultad también reside en el hecho de que no hay límite claro de división o segmentación de los sonidos percibidos en el habla. Ambos aspectos, de relación compleja entre la señal acústica del mensaje y el componente fonológico y de alteración cuando los fonemas son segmentados, explican la dificultad de la tarea de segmentación en fonemas. Por ello, hay autores que sostienen que la segmentación fonológica se va consolidando cuando se captan las correspondencias entre el lenguaje oral y el escrito: las letras permitirían apoyar la segmentación del habla en fonemas (Vernon y Ferreiro, 1999). En definitiva, se defiende que la adquisición de la escritura ayuda a los niños a reconocer y trabajar sobre los segmentos que descomponen las palabras (Defior, 1994).

Por otro lado, autores como Bryant y Bradley $(1993,1998)$ sostienen que las experiencias de los 
niños con las unidades rítmicas de las palabras son fundamentales en el desarrollo de la conciencia fonológica y en el acceso a la lectura. En sus estudios demostraron que los preescolares son capaces de detectar rimas con gran facilidad. Para los autores, esas experiencias realizadas mucho antes de la adquisición de la lectura y de la escritura, constituyen precursores importantes del desarrollo de la conciencia fonémica y del éxito en la lectura. Plantean que trabajar con los sonidos que riman es importante porque ayudan a los niños a tener en cuenta las secuencias de letras, ya que las palabras que riman suelen tener letras en común (Bryant y Goswami, 1987). En definitiva, una serie de investigaciones ha demostrado que existe una gran sensibilidad infantil a la rima y a la aliteración. Dicha sensibilidad aparece a una edad muy temprana, antes de la instrucción formal. Para muchos autores, este hecho es un predictor del éxito en la lectura y la escritura.

Sin entrar en el debate de que la sensibilidad a la rima exige unas habilidades comparables a otras segmentaciones, lo cierto es que la gran mayoría de tareas para detectar dicha sensibilidad no exige a los niños una reflexión metalingüística más analítica de otras unidades más sutiles, como el fonema. Por lo tanto, no queda demostrado que los niños, a partir de una sensibilidad a la rima, lleguen a una segmentación fonémica, a pesar de que está comprobado que hay una relación significativa entre habilidades rítmicas y buenos resultados en lectura en diferentes lenguas.

En una posición distinta a la anterior, Morais (1994) y sus colaboradores mostraron que existe una estrecha relación entre conocimiento fonológico y habilidades lectoras y de escritura. Este autor defiende la idea de que la habilidad en detectar y producir rimas no presupone que los niños hayan adquirido la capacidad de analizar la sílaba en sus unidades intrasilábicas, es decir, el ataque (que es la consonante o grupo consonántico que precede a la vocal) y la rima (vocal que constituye el núcleo). La habilidad de analizar la sílaba en unidades intrasilábicas ha sido detectada en muchos sujetos de lengua inglesa. Sin embargo, cabe decir que una serie de investigaciones relacionadas con la conciencia metalingüística con niños de lengua románica demuestran que sus resultados no coinciden con los datos obtenidos en infantes de lengua inglesa (Cardoso-Martins, 1995; Vernon, 1998; Barrera, 2000). La mayoría de los estudios en inglés tienden a generalizar los resultados encontrados y en la actualidad existen datos que demuestran la importancia de la naturaleza de la lengua en el ámbito de este tema (Defior, 1994; Vernon, 1998).

Puesto que se trata de niños pequeños, el contexto de juego de palabras parece ser muy adecuado para estimular reflexiones sobre el lenguaje y fomentar la conciencia fonológica, además de ejercer un gran atractivo entre los infantes. Se supone que los niños cuando juegan con la lengua también juegan con su estructura (Cook, 2000). De esta manera, resulta oportuno presentar este tipo de texto en actividades que enfocan los aspectos formales y lexicales del lenguaje. Utilizamos los juegos de palabras para garantizar la centración sobre la estructura fonológica y morfológica de las palabras, y no en su relación de significación.

El presente estudio tiene como objetivo principal estudiar la sensibilidad a los aspectos sonoros del lenguaje en relación con los procesos de adquisición de la escritura en niños preescolares brasileños. Se pretende plantear una perspectiva que tenga en cuenta: los niveles de conceptualización de la escritura y no sólo la lectura, las interacciones entre oralidad y escritura, diferentes aspectos formales (fonológicos, morfológicos y lexicales) y no sólo fonológicos o semánticos, otras lenguas que no sean el inglés, en este caso el portugués y contextos de juego de palabras.

\section{Método}

En este estudio fueron entrevistados 40 niños preescolares provenientes de dos escuelas de titularidad privada de la ciudad de Salvador de Bahía, en Brasil, correspondientes a los cursos de Párvulos 4 (4 años), Párvulos 5 (5 años) y Primero (6 años). El rango de 
edad de los participantes fue de 4:6 a 6:6 (media $=$ 5:5), siendo 17 niños y 23 niñas. Todos los participantes tenían el portugués como lengua materna.

Las entrevistas fueron realizadas en las escuelas, con una duración aproximada de 30 minutos. Las sesiones fueron registradas en cintas magnetofónicas y en folios por la propia entrevistadora. El diseño metodológico consistió en una tarea previa de nivel de conceptualización de la escritura, seguidas de la Tarea 1 (Rima) y de la Tarea 2 (Segmentación en eco). En la tarea de evaluación de nivel de escritura, se pidió a cada participante que escribiese cuatro palabras del mismo campo semántico (nombres de animales) y una frase. Una vez escrita cada una de las palabras, se pidió a los niños que se las leyera en voz alta, señalando con el dedo lo que habían escrito. Los niveles de escritura fueron analizados siguiendo los estudios de Ferreiro y Teberosky (1979) y Ferreiro (1986). Las respuestas infantiles fueron categorizadas en cinco grupos que corresponden a formas de conceptualizar lo escrito y su relación con la oralidad: 1. Presilábico, 2. Silábico estricto sin valor sonoro, 3. Silábico estricto con valor sonoro, 4. Silábico alfabético y 5 . Alfabético.

En función del rendimiento de los niños con respecto a la correspondencia grafema/fonema, decidimos agrupar los seis niveles en tres grupos. Un primer grupo sin ningún tipo de correspondencia fonográfica o con algún procedimiento silábico, pero sin valor sonoro convencional; un segundo grupo con un nivel de correspondencia silábica, pero con valor sonoro convencional, y finalmente un tercer grupo con correspondencia alfabética exhaustiva. Tales agrupaciones quedaron de la siguiente forma: Grupo 1= Presilábicos y silábicos sin valor sonoro; Grupo 2= Silábicos estrictos con valor sonoro y silábicos alfabéticos y Grupo 3= Alfabéticos. Una vez realizada la tarea de evaluación de nivel de escritura, se inició la entrevista. A continuación se explicarán los procedimientos utilizados en las tareas.

Tarea de Rima (Tarea 1) “¿Qué se parece aquí?" En la modalidad oral se instaba a los participantes: "¿Vamos a jugar? fíjate bien como es este juego".
En ese momento se empezaba a leer en voz alta y muy despacio versos con rima del fragmento de poesía:

Frase 1: O pato ganhou um sapato e foi fazer retrato (fragmento de un poema de Mário Quintana)

Frase 2: $O$ macaco retratista era um grande artista malabarista (fragmento de un poema de Mário Quintana)

Frase 3: Na casa azulzinha havia uma menininha com uma bonequinha

Versos utilizados en la Tarea 1:

Después se les preguntaba: “'hay nombres que se parecen?, ¿cuáles se parecen más aquí?”. En los casos que los niños no contestaran, se volvía a repetir el verso. En los demás versos fue utilizado el mismo procedimiento. Posteriormente, en la modalidad escrita, se presentaban tarjetas impresas con las palabras rimadas de los versos dispuestos en la mesa: "ahora te enseño estas tarjetas pato, zapato y retrato", ¿crees que hay algo que sea parecido aquí?, ¿qué se parece más en estos tres nombres? El mismo procedimiento fue utilizado en las demás palabras rimadas de las dos frases restantes.

Tarea de Segmentación en Eco (Tarea 2) “¿Qué ha dicho el eco?"- En esta tarea se le comunicaba a los niños: "Ahora vamos a jugar a otra cosa. Escucha esto y fijate bien en lo que hacen". A continuación se les leía esta una nueva poesía:

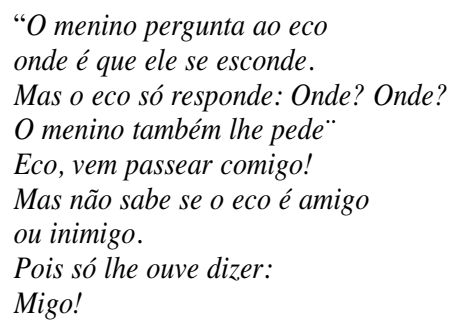

Cecília Meireles

y se les preguntaba: ¿hay nombres que se parecen aquí?, ¿cuáles?, ¿sabes qué es un eco? En el caso de que no lo supieran, la experimentadora se lo explicaba, dando algunos ejemplos con una grabación simulada de una voz de un niño y otra de su eco. La repetición del eco en los nuevos estímulos, estuvo relacionda a palabras con flexión para ver si tal repetición recaía sobre fonema, sílaba o morfe- 
ma, todos en posición final en palabras de distintas estructuras.

Después de darles el modelo se enseñaba dos títeres y se les decía:

\footnotetext{
"Te presento ahora estos dos muñecos, el primero es el niño y lo haré yo, y el segundo es el eco, y lo harás tú. El niño (yo) dirá unos nombres y el eco (tú) dirá otros, ¿vale? Acuérdate de que el eco no repite todo, sólo un trozo".
}

Inmediatamente después, la entrevistadora representando al niño decía frases poniendo énfasis en la última palabra. En la modalidad escrita, se decía: "el niño gritó este nombre y lo dejó así en una roca”. En este momento se leía una frase escribiendo la última palabra con las letras móviles y luego les preguntaba: ¿qué dirá el eco? En el caso de que los niños no contestaran se les decía: ¿tú que eres el eco, qué repetirías?, ¿qué trocito le quitarías? Los estímulos en portugués utilizados em ambas modalidades fueron: Eco vem comprar pão, Eco vem descansar, Eco para de ser mau, Eco vem chutar a bolinha, Eco vem agora comer, Eco vem comer um doce gostoso.

\section{Resultados}

Debido a las características de las variables dependientes y el tamaño de la muestra se aplicaron pruebas no paramétricas en ambas tareas. Dichas pruebas fueron la $\mathrm{H}$ de Kruskal-Wallis en la comparación de los tres grupos independientes de niveles de escritura, la U de Mann-Whitney para comparar los niveles de escritura a posteriori y la T de Wilcoxon para comparar las modalidades oral y escrita. Los criterios de codificación de las tareas de rima (Tarea 1) y de segmentación en eco (Tarea 2) estuvieron basados en el tipo de trabajo que realizaban los participantes: la extracción de palabras (en el caso de la tarea de rima) o la segmentación (en fonema, sílaba o morfema en ambas tareas) fueron clasificadas como respuesta que considera aspectos de tipo formal. Tanto para la tarea de rima como para la tarea de segmentación en eco se analizaron separadamente las respuestas con segmentación de las respuestas sin segmentación. Debido a que existieron varios estímulos en cada tarea se realizó una suma de los mismos, con esto se obtuvo una puntuación total por modalidad.

En la Tabla 1 se presentan los resultados de la primera pregunta para el análisis estadístico de la tarea de rima: ¿el niño hizo el trabajo de encontrar rimas? En la modalidad oral no se encontraron respuestas con extracción del segmento rimado, por lo que no nos pareció necesario exponerlos aquí.

Tabla 1: Prueba de comparación de la Tarea de Rima teniendo en cuenta los grupos de niveles de escritura: Modalidad escrita

\begin{tabular}{|c|c|c|c|}
\hline Grupos & $\begin{array}{l}\text { Media } \\
\text { del } \\
\text { Rango }\end{array}$ & $\begin{array}{c}\text { H de K. } \\
\text { Wallis }\end{array}$ & $\begin{array}{c}\text { Contrastes a posteriori } \\
\text { U de Mann-Whitney } \\
\text { ( }{ }^{\mathrm{a} c o m p a r a c i o n e s)}\end{array}$ \\
\hline
\end{tabular}

\begin{tabular}{|c|c|c|c|}
\hline Grupo 1 & 14.09 & & Grupo 1 y 2 z $=2.706$ y p \\
\hline Grupo 2 & 26.55 & $\chi(2)^{2}=9.067$ & Grupo 1 y $3 z=2.279$ y \\
\hline Grupo 3 & 23.50 & $p=0.011$ & Grupo 2 y $3 \mathrm{z}=0.695$ y $\mathrm{p}$ \\
\hline \\
\hline & & & \\
\hline \multicolumn{4}{|c|}{$\begin{array}{l}\text { Grupo } 1 \text { - Presil. y sil. sin valor sonoro convencional }(n=16) \\
\text { Grupo } 2 \text { - Sil. con valor sonoro convencional y sil. alfabético }(n=10)\end{array}$} \\
\hline \multicolumn{4}{|c|}{ Grupo 3 - Alfabético $(n=14)$} \\
\hline
\end{tabular}

Sin embargo, en la modalidad escrita se pueden observar diferencias estadísticamente significativas, $\left(\mathrm{x}^{2}(2)=9.067 \mathrm{y} \mathrm{p}=0.011\right)$. Con la prueba $\mathrm{U} \mathrm{de}$ Mann-Whitney en los contrastes a posteriori se puede ver que dichas diferencias aparecieron entre el grupo 1 (presil. y sil. sin vsc) y el grupo 2 (sil. con vsc y sil. alf.), ( $\mathrm{z}=2.706$ y $\mathrm{p}=0.007)$. También se puede observar que se encontraron diferencias estadísticamente significativas entre el grupo 1 (presil. y sil. sin vsc) y el grupo 3 (alfabético), $(\mathrm{z}=2.279 \mathrm{y}$ $\mathrm{p}=0,028)$. Con los resultados obtenidos se puede concluir que los niños del grupo 1 (presil. y sil. sin vsc) segmentaron menos la rima en las palabras con respecto a los niños del grupo 2 (sil. con vsc y sil. alf.) y del grupo 3 (alfabético); mientras que los participantes de éstos últimos realizaron un trabajo de segmentación de rimas, en la modalidad escrita, sin diferencias de procedimientos.

La Tabla 2 se refiere a los resultados de la segunda pregunta para el análisis estadístico de la tarea de rima: ¿el niño hizo un trabajo de extracción? En la modalidad escrita no se aplicó la prueba de contras- 
tes a posteriori puesto que no aparecieron diferencias estadísticamente significativas, $\left(\mathrm{x}^{2}(2)=4.932 \mathrm{y}\right.$ $\mathrm{p}=0.085)$. En cambio, en la modalidad oral se produjeron tales diferencias, $\left(\mathrm{x}^{2}(2)=11.790 \mathrm{y} \mathrm{p}=0.003\right)$. Estas diferencias fueron encontradas entre el grupo 1 (presil. y sil. sin vsc) y el grupo 2 (sil. con vsc y sil. alf.), $(\mathrm{z}=2.336$ y $\mathrm{p}=0.027)$ y entre el grupo 1 (presil. y sil. sin vsc) y el grupo 3 (alfabético), $(\mathrm{z}=3.070$ y $\mathrm{p}=0.040)$.

Tabla 2. Prueba de comparación de la Tarea de Rima teniendo en cuenta los grupos de niveles de escritura

\begin{tabular}{cccc}
\hline Grupos & Media & & \\
& del & Hde K. & Contrastes a posteriori \\
Rango & Wallis & Ude Mann-Whitney \\
& & ('comparaciones)
\end{tabular}

Modalidad Escrita

\begin{tabular}{|c|c|c|c|}
\hline 1 & 17.75 & & 1 \\
\hline & 20. & $\chi(2)^{2}=4.93$ & 0.019 \\
\hline ut & 24.00 & $p=0.085$ & $=1.183 \mathrm{y}$ \\
\hline
\end{tabular}

Modalidad Oral

Grupo $1 \quad 13.66 \quad$ Grupo 1 y $2 \mathrm{z}=2.33$ y $\mathbf{p}=\mathbf{0 . 0 2 7}$

Grupo $2 \quad 23.10 \quad \chi(2)^{2}=1.79 \quad$ Grupo 1 y $3 \mathrm{z}=3.070$ y $\mathbf{p}=\mathbf{0 . 0 4 0}$

Grupo $326.46 \quad \mathbf{p}=\mathbf{0 . 0 0 3} \quad$ Grupo 2 y $3 \mathrm{z}=1.241$ y $\mathrm{p}=0.371$

Nota. Los grupos se refieren a los niveles de escritura que son los siguientes:

Grupo 1 - Presil. y sil. sin valor sonoro convencional $(n=16)$.

Grupo 2 - Sil. con valor sonoro convencional y sil. alfabético $(\mathrm{n}=10)$.

Grupo 3 - Alfabético $(n=14)$

$\mathrm{a}_{\text {comparaciones de los grupos de niveles de escritura }}$

Se puede concluir a partir de los resultados, que los niños del grupo 1 (presil. y sil. sin vsc) valoraron de distinta forma el trabajo de extracción de palabras en las frases con respecto a los participantes de los demás grupos. Estos niños del grupo 1 realizaron con menos rendimiento el trabajo de extracción que los participantes del grupo 2 (sil. con vsc y sil. alf.) y del grupo 3 (alfabético). Sin embargo, estos dos últimos grupos realizaron extracciones sin diferencias estadísticamente significativas de procedimientos.

En la Tabla 3 se presentan los resultados de la primera pregunta del análisis estadístico de la Tarea de Segmentación en Eco, dicha pregunta fue: ¿el niño hizo un trabajo de segmentación? En la modalidad escrita se produjeron diferencias estadísticamente significativas, $\left(\mathrm{x}^{2}(2)=11.380 \mathrm{y} \mathrm{p}=0.003\right)$. Se puede observar que tales diferencias aparecieron entre el grupo 1 (presil. y sil. sin vsc) y el grupo 3 (alfabético), $(\mathrm{z}=2.996$ y p=0.019). Por otro lado, también se produjeron diferencias estadísticamente significativas en el oral, $\left(x^{2}(2)=10.741\right.$ y $\left.p=0.005\right)$. Se puede ver que estas diferencias también fueron encontradas entre el grupo 1 (presil. y sil. sin vsc) y el grupo 3 (alfabético), $(\mathrm{z}=3.115$ y $\mathrm{p}=0.004)$.

Tabla 3. Prueba de comparación de la Tarea de Segmentación en Eco teniendo en cuenta los grupos de niveles de escritura

\begin{tabular}{cccc}
\hline Grupos & Media & & \\
& del & Hde K. & Contrastes a posteriori \\
& Rango & Wallis & Ude Mann-Whitney \\
& & & ('acomparaciones)
\end{tabular}

Modalidad Escrita

\begin{tabular}{llcc}
\hline Grupo 1 & 15.00 & & Grupo 1 y $2 \mathrm{z}=1.991$ y $\mathrm{p}=0.097$ \\
Grupo 2 & 23.00 & $\chi(2)^{2}=11.380$ & Grupo 1 y 3 $\mathrm{z}=2.996$ y $\mathbf{~}=\mathbf{0 . 0 1 9}$ \\
Grupo 3 & 25.00 & $\mathbf{p}=\mathbf{0 . 0 0 3}$ & Grupo 2 y $3 \mathrm{z}=1.183$ y $\mathrm{p}=0.709$
\end{tabular}

\begin{tabular}{|c|c|c|c|}
\hline \multicolumn{4}{|c|}{ Modalidad Oral } \\
\hline Grupo 1 & 14.31 & & Grupo 1 y $2 \mathrm{z}=1.767$ y $\mathrm{p}=0.097$ \\
\hline Grupo 2 & 21.30 & $\chi(2)^{2}=10.741$ & Grupo 1 y $3 \mathrm{z}=3.115$ y $\mathbf{p}=\mathbf{0 . 0 0 4}$ \\
\hline Grupo 3 & 27.00 & $p=0.005$ & Grupo 2 y $3 \mathrm{z}=1.752$ y $\mathrm{p}=0.172$ \\
\hline \multicolumn{4}{|c|}{$\begin{array}{l}\text { Nota. Los grupos se refieren a los niveles de escritura que son los siguientes: } \\
\text { Grupo } 1 \text { - Presil. y sil. sin valor sonoro convencional }(\mathrm{n}=16) \text {. } \\
\text { Grupo } 2 \text { - Sil. con valor sonoro convencional y sil. alfabético }(\mathrm{n}=10) \text {. } \\
\text { Grupo } 3 \text { - Alfabético }(\mathrm{n}=14) \\
\mathrm{a}_{\text {comparaciones de los grupos de niveles de escritura }}\end{array}$} \\
\hline
\end{tabular}

A partir de estos resultados se pueden concluir que, en ambas modalidades, los participantes del grupo 1 (presil. y sil. sin vsc) realizaron menos segmentaciones que los participantes del grupo 3 (alfabético) en los estímulos. Entre los participantes del grupo 2 (sil. con vsc y sil. alf.) y del grupo 3 (alfabético) no fueron encontradas diferencias estadísticamente significativas en ambas modalidades. Se hicieron frecuencias de cuántos participantes por grupo de nivel de escritura realizaron segmentaciones por número de estímulos. Recordemos que fueron presentados un total de seis estímulos por modalidad.

En la Tabla 4 se pueden ver los resultados de la distribución de frecuencias en la modalidad escrita. En dicha modalidad, los participantes de mayor 
nivel alfabético de escritura (grupo 3- alfabético) segmentaron más estímulos que los participantes de los demás grupos, con un $100 \%$ de segmentaciones en todos los estímulos. En cambio, los niños del grupo 2 (sil. con vsc y sil. alf) segmentaron un total de $90 \%$ de los seis estímulos frente a los participantes del grupo 1 (presil. y sil. sin vsc) que realizaron un $50 \%$ de segmentaciones en el mismo número de estímulos.

Tabla 4. Distribución de frecuencias de cuántos participantes por grupo realizaron segmentaciones por número de estímulo

Modalidad Oral

Grupos Número de Estímulos

\begin{tabular}{lccccccc} 
& 0 & 1 & 2 & 3 & 4 & 5 & 6 \\
\hline Grupo 1 & $6.3 \%$ & $6.2 \%$ & $12.5 \%$ & -- & $25 \%$ & -- & $50 \%$ \\
Grupo 2 & -- & -- & -- & $10 \%$ & -- & -- & $90 \%$ \\
Grupo 3 & -- & -- & -- & -- & -- & -- & $100 \%$ \\
\hline
\end{tabular}

Nota. Los grupos se refieren a los niveles de escritura que son los siguientes:

Grupo 1 - Presil. y sil. sin valor sonoro convencional $(n=16)$.

Grupo 2 - Sil. con valor sonoro convencional y sil. alfabético $(\mathrm{n}=10)$.

Grupo 3 - Alfabético $(\mathrm{n}=14)$

En la modalidad oral también se puede ver que a mayor nivel de escritura, más segmentaciones en un mayor número de estímulos (Tabla 5). Los participantes del grupo 3 (alfabético) realizaron un 85.7\% de segmentaciones en todos los seis estímulos, frente a un $50 \%$ de segmentaciones de los participantes del grupo 2 (sil. con vsc y sil. alf) y de un $31.3 \%$ de segmentaciones de los participantes del grupo 1(presil. y sil. sin vsc). Con estos datos podemos afirmar que los participantes de mayor nivel de escritura

Tabla 5. Distribución de frecuencias de cuántos participantes por grupo realizaron segmentaciones por número de estímulo

Modalidad Escrita

\begin{tabular}{lccccccc}
\hline Grupos & \multicolumn{7}{c}{ Número de Estímulos } \\
& 0 & 1 & 2 & 3 & 4 & 5 & 6 \\
\hline Grupo 1 & $50 \%$ & -- & $6.2 \%$ & -- & -- & $12.5 \%$ & $31.3 \%$ \\
Grupo 2 & -- & $20 \%$ & $10 \%$ & -- & -- & $20.0 \%$ & $50.0 \%$ \\
Grupo 3 & -- & $7.1 \%$ & -- & -- & $7.1 \%$ & -- & $85.8 \%$ \\
\hline
\end{tabular}

Nota. Los grupos se refieren a los niveles de escritura que son los siguientes: Grupo 1 - Presil. y sil. sin valor sonoro convencional $(n=16)$.

Grupo 2 - Sil. con valor sonoro convencional y sil. alfabético $(\mathrm{n}=10)$.

Grupo 3 - Alfabético $(\mathrm{n}=14)$. realizaron más segmentaciones en un mayor número de estímulos. El procedimiento de segmentar más estímulos se ha evidenciado en ambas modalidades. Sin embargo, como vimos en las pruebas estadísticas, aparecieron diferencias significativas solamente entre el grupo $1 \mathrm{y}$ el grupo 3.

También se aplicó la T de Wilcoxon en la comparación entre la modalidad oral y escrita de las tareas de rima (Tarea 1) y de segmentación en eco (Tarea 2). Los resultados de la Tabla 6 revelan que existieron diferencias estadísticamente significativas en las modalidades oral y escrita de ambas tareas. Analizando los resultados de la tarea de rima se puede afirmar que los niños realizaron mejor la modalidad escrita que la modalidad oral, $(\mathrm{z}=4.939 \mathrm{y}$ $\mathrm{p}<0.001)$. Por otro lado, en la tarea de segmentación en eco también aparecieron diferencias estadísticamente significativas, los niños realizaron más segmentaciones en la modalidad escrita que en la modalidad oral, $(\mathrm{z}=2.812$ y $\mathrm{p}=0.005)$.

Tabla 6. Comparación entre las modalidades ral y escrita de las tareas de rima y de segmentación

\begin{tabular}{lcc}
\hline Tareas & Modalidad & Tde Wilcoxon \\
\hline 1 & oral/ escrita & $\mathrm{z}=4.939 \mathbf{p}<\mathbf{0 . 0 0 1}$ \\
2 & oral/ escrita & $\mathrm{z}=2.812 \mathbf{p}=\mathbf{0 . 0 0 5}$ \\
\hline
\end{tabular}

En definitiva, los resultados encontrados en la Tarea de Rima (Tarea 1) y de Segmentación en Eco (Tarea 2) nos confirman la hipótesis que postula que en tareas de segmentación existe un fuerte eslabón entre procesos metalingüísticos de segmentación de palabras y uso de la escritura en los diseños metodológicos.

\section{Discusión}

Los resultados obtenidos en las tareas aquí diseñadas nos permiten hacer las consideraciones que se expondrán a continuación. En la Tarea de Rima (Tarea 1), la consigna "qué se parece más" fue respondida con una extracción de palabras en la 
modalidad oral y no fueron encontradas extracción de sílaba o de morfema en esta modalidad. Creemos que este tipo de extracción más analítica requiere un trabajo cognitivo más elaborado. El hecho de que ningún niño haya realizado otro tipo de segmentación que no fuese la segmentación de palabras nos da información para afirmar que la consigna y los estímulos en frases orales fueron interpretados como una extracción de la palabra rimada y no de la rima en sí misma.

En efecto, los resultados encontrados corroboran la afirmación de que la modalidad de presentación de la tarea influye en la resolución de la misma. Los estímulos escritos, de cierta forma, facilitaron la extracción del segmento rimado, puesto que algunos de los niños de menor nivel de escritura identificaron la rima con la estrategia gráfica o visual. Independientemente de la estrategia utilizada en la tarea de rimas, los análisis estadísticos revelaron que los niños realizaron mejor la modalidad escrita. Los participantes del grupo 1 (presil. y sil. sin vsc) con respecto a los demás grupos, realizaron la tarea con menos éxito. Estos niños del grupo 1 muchas veces señalaban letras aisladas dejando claro que se trataba de extracciones sin ningún tipo de estrategia. Este procedimiento no fue categorizado como un trabajo de segmentación. No obstante, la gran mayoría de los niños identificaron la rima o parte de ella (sílabas o morfemas) en los estímulos presentados. Como era de esperar, ningún participante dio respuestas semánticas en la modalidad escrita. También en la modalidad oral el nivel de escritura fue otro factor que estuvo relacionado con la tarea. En general, los participantes del grupo 1 (presil. y sil. sin vsc) realizaron con menos éxito el trabajo en dicha modalidad.

A nivel general, solamente un $12 \%$ de los participantes del grupo 2 (sil. con vsc y sil. alf) y un $8 \%$ de los participantes del grupo 3 (alfabético) dieron algún tipo de respuesta semántico-lexical más que de extracción de rima. En cambio un 38\% de los niños del grupo 1 (presil. y sil. sin vsc), en al menos alguno de los estímulos orales, contestaron con ese tipo de respuesta. También hubo casos de niños de nivel presilábico que lograron detectar la rima en al menos una de las frases. Es importante destacar que la gran mayoría de los niños pertenecientes al grupo 3 (alfabético) lograron detectar al menos dos de las tres palabras rimadas en las frases en la modalidad oral.

Ya en la tarea de segmentación en eco (Tarea 2), en el desarrollo de la investigación, hemos podido constatar que se produjeron algunas diferencias de procedimientos según el estímulo, de manera que cuestionamos qué tipo de segmentación darían en el juego de "segmentar en eco". De forma global, dos tipos de respuestas fueron encontradas: 1) Respuestas con segmentación silábica, mayoritarias en ambas modalidades (oral y escrita) y 2) Respuestas con omisión de letra, mayoritarias en la modalidad escrita. La categoría "sin segmentación" obtuvo un considerado número de respuestas, sobre todo en la modalidad oral: un $32.5 \%$. Como era previsto, las respuestas con relaciones semánticas fueron escasas prevaleciendo repeticiones o respuestas de tipo "no lo sé". A raíz de los resultados podemos concluir que los procedimientos en la tarea de segmentación en eco se distribuyeron de forma que:

Segmentación silábica: fue mayoritaria, se distribuyó en todos los estímulos, pero fue más empleada en la modalidad escrita.

Segmentación morfológica: fue más alta en los estímulos con morfemas más destacados, como por ejemplo bolinha que obtuvo un $12,5 \%$ de este tipo de segmentación en la modalidad escrita y un $25 \%$ en la modalidad oral. La segmentación morfémica fue más usada en la modalidad oral que en la escrita.

Segmentación fonológica: fue escasa en esta tarea, las pocas respuestas se concentraron en los monosílabos y como segmentación de letra. Este tipo de segmentación fue más empleada en la modalidad escrita.

Omisión de letra o fonema inicial: fue más utilizada en los monosílabos ( $p a ̃ o, m a u)$. En la modalidad escrita este tipo de trabajo fue usado por un mayor número de participantes.

Sin segmentación: no realizar ninguna segmentación fue un tipo de procedimiento más empleado en la modalidad oral $(32.5 \%)$ que en la escrita $(12,1 \%)$. 
En la modalidad oral los participantes presentaron más dificultades en segmentar los monosílabos tendiendo a repetir el estímulo. Cabe destacar que el nivel de escritura no estuvo relacionado con segmentaciones más analíticas, la segmentación silábica fue predominante incluso para los alfabéticos, lo que nos llevó a cuestionar la influencia de las características de la tarea en el tipo de segmentación realizada.

En general, los procedimientos fueron de segmentación silábica en la mayoría de los estímulos. Sin embargo, la segmentación morfémica se pudo ver en las palabras con la estructura morfémica más destacada; y la omisión de grafema o fonema inicial fueron más empleadas en los monosílabos y bisílabo. El estímulo ejerció cierta influencia, de modo que en los monosílabos las segmentaciones orales resultaron más difíciles y en algunos de los trisílabos se pudo ver segmentaciones morfémicas. También en los monosílabos el trabajo de omisión de letra o fonema inicial resultó más fácil que en los estímulos de tres o cuatro sílabas. Además, la modalidad de presentación de la tarea influyó en las respuestas: en general, se produjeron más segmentaciones en la modalidad escrita. Otra conclusión es que las características de la tarea llevaron a un trabajo básicamente formal y los niños de nivel silábico alfabético y alfabético tendieron a segmentar las palabras, sin embargo cuando les resultaba difícil o repetían el estímulo o no contestaban. No obstante, no podemos afirmar del todo que la estructura de la palabra juega un importante papel en el tipo de segmentación sobre la misma. Habría que utilizar en futuras investigaciones una variable que controlase la estructura y longitud de las palabras en un diseño metodológico que presentase un mayor número de palabras de estructura interna semejante.

\section{Conclusiones}

En este artículo hemos podido ver los resultados obtenidos en tareas de rima y segmentación realizadas con niños preescolares de Brasil. Los resultados de la tarea de rima revelaron que los niños interpre- taron la modalidad oral como una extracción de la palabra rimada y no de la rima en sí misma. Sin embargo han podido identificar y segmentar la rima en la modalidad escrita. Las diferencias se encontraron entre el grupo 1 (presil. y sil. sin vsc) y el grupo 2 (sil. con vsc y sil. alf.), y entre el grupo 1 (presil. y sil. sin vsc) y el grupo 3 (alfabético). Para la pregunta que se refiere a la extracción, hemos podido ver que aparecieron diferencias estadísticamente significativas entre el grupo 1 (presil. y sil. sin vsc) y el grupo 2 (sil. con vsc y sil. alf.), y además entre el grupo 1 (presil. y sil. sin vsc) y el grupo 3 (alfabético).

Para concluir recordemos que el nivel de escritura influyó en la resolución, de manera que los niños de menor nivel de escritura realizaron menos extracciones de palabras y del segmento rimado que los demás participantes. Los resultados de la tarea de segmentación en eco fueron similares a los obtenidos en la tarea de rima. En general, los niños de menor nivel de escritura realizaron con menos rendimiento la tarea, es decir hicieron menos segmentaciones en ambas modalidades. Una vez más los niños presilábicos y silábicos estrictos sin valor sonoro (grupo 1) presentaron más dificultades en la resolución, en este caso, en segmentar palabras. No obstante, los más avanzados en la conceptualización de la lengua escrita presentaron menos dificultades en segmentar los estímulos. Este dato es un indicador de que el desarrollo de la escritura presenta una importante relación con este tipo de tarea enfocada en los aspectos sonoros del lenguaje.

$\mathrm{Al}$ igual que en la tarea de rima hemos podido constatar que la modalidad de presentación influyó en la resolución de la tarea de segentación. También hemos podido ver que el trabajo de segmentación en la modalidad escrita siguió siendo el más alto. Con la prueba $\mathrm{T}$ de Wilcoxon se confirmó que los participantes realizaron mejor la modalidad escrita de la tarea. Este dato nos lleva a concluir que: 1) La modalidad de presentación de la tarea es una importante variable a ser considerada en este tipo de tarea y 2) Las palabras escritas obtuvieron un mayor número de segmentaciones. 
Con los resultados estadísticos presentados no se pudo ver que tipo de segmentaciones realizaron los niños (morfémica, silábica, etc.). Sin embargo, en el análisis por estímulo, pudimos constatar que la segmentación silábica fue la mayoritaria. Como lo ocurrido en la tarea de rima, el diseño de la tarea parece haber jugado un importante papel no orientando a segmentaciones más analíticas.

Otro punto destacable de la tarea de segmentación en eco ha sido las características de los estímulos. En el análisis individual de los mismos hemos podido ver que el número de sílabas influyó en el tipo de segmentación, de modo que el trabajo de omisión de letra o fonema inicial estuvo más concentrado en los monosílabos y en el único bisílabo. Por otro lado, los trisílabos con morfemas más destacados recibieron un considerado número de segmentación morfémica.

A partir de los resultados presentados se puede concluir que el nivel de escritura influyó en la resolución de la tarea, así como la modalidad de presentación. Estudios anteriores (Ferreiro, 1996; Vernon y Ferreiro, 1992) ya habían demostrado la importancia de añadir el nivel de escritura como variable, y no solamente la edad del participante, y diseños que se tuviera en cuenta la modalidad escrita en tareas metalingüísticas de conocimiento fonológico. Nuestra investigación corrobora estas dos afirmaciones tal como se pudo ver en los resultados encontrados.

Vimos que muchos de los niños fueron sensibles a la forma de los estímulos demostrando habilidades para segmentar frases y palabras en las tareas propuestas. Con los resultados obtenidos hemos podido observar que la presencia de la escritura (tarjetas impresas o letras móviles) produjo un incremento de respuestas más finas.

No obstante, mismo con la presencia de la escritura el tipo de segmentación más accesible fue la segmentación de palabras o del segmento rimado (Tarea de Rima) y la segmentación silábica (Tarea de Segmentación en Eco). Es decir, la segmentación fonémica fue minoritaria y se concentró, en su mayoría, en los casos de omisión de fonema/letras iniciales en la tarea de segmentación en eco. En la tarea de segmentación, aunque el modelo previo simulando a un eco daba como ejemplos segmentaciones silábicas, morfémicas y fonémicas, prevaleció la segmentación silábica. Este dato corrobora los resultados obtenidos en diferentes estudios que afirman que la habilidad de tratar las sílabas es la de más fácil acceso y anterior a la segmentación fonémica (Morais, 1991; Jiménez, 1992 y Defior, 1996).

Por otro lado, nuestros datos ponen de manifiesto la afirmación de que la comprensión del sistema alfabético lleva a la adquisición de la conciencia fonémica (Alegría, 1993; Morais, 1991). Con los resultados encontrados podemos afirmar que la realización de segmentaciones exhaustivas no resulta inmediatamente del conocimiento de la escritura alfabética, al menos no de forma automática y exclusiva. Este dato también fue encontrado por Vernon (1998) en un trabajo con niños mexicanos. Eso es un indicador de que no todos los tipos de segmentación presentan el mismo grado de dificultad (Defior, 1996). Como vimos, en la tarea de rima no pareció que las características de los estímulos hayan influido en la resolución de la tarea. En cambio, en la tarea de segmentación en eco vimos que no todas las palabras se prestaron a un mismo tratamiento segmental aunque, en general, prevalecieron las segmentaciones silábicas.

Como hemos dicho anteriormente, hay una abundancia de estudios sobre la conciencia fonológica con niños de lengua inglesa. A raíz de estos estudios, se hicieron generalizaciones en resultados que medían dicha conciencia, de manera que se ha dado mucho énfasis a la importancia de las unidades intrasilábicas ataque (onset) y rima (rime). La importancia de estas unidades intrasilábicas no ha sido demostrada en niños de lenguas castellana y portuguesa (Vernon, 1998; Carrillo, 1994 y Cardoso-Martin, 1995, Ribeiro y Teberosky, 2007). Por otro lado, también presentamos resultados en los que la segmentación silábica fue más frecuente que la segmentación morfémica incluso en los estímulos que presentaban estructura morfémica destacada y usual en portugués.

En conclusión, creemos que no todas las tareas de 
conciencia fonológica llevan a segmentaciones exhaustivas, es decir a la segmentación fonémica. Las características de las tareas aquí diseñadas no parecen haber ayudado de manera explícita a que los participantes llegasen a segmentaciones más analíticas. En los diversos estudios encontrados en la literatura sobre este tema se puede constatar la diferencia de resultados obtenidos en los distintos diseños metodológicos de tareas que evalúan la conciencia fonológica. En este sentido nuestros datos apoyan a los de Defior (1996, p.56) cuando afirma que "existen grandes diferencias en las demandas cognitivas que plantean las diversas tareas fonológicas y, por lo tanto, también en la dificultad que conllevan para su realización". Además, como hemos visto, entre las segmentaciones realizadas no fueron encontradas las de tipo onset como la gran mayoría de los trabajos realizados en lengua inglesa revelan, lo cual corrobora la afirmación que no se puede generalizar resultados en este tema de estudio.

Finalmente podemos afirmar que la adquisición de la escritura influye en el conocimiento fonológico dado que a mayor nivel de escritura, más extracciones y segmentaciones fueron realizadas.

\section{Referencias}

Alegría, J. (1993). Análisis segmental y adquisición de la lectura a propósito de lo que el niño sabe y de lo que ignora cuando comienza a enseñarle a leer. Lenguaje y Comunicación, 8, 88-96.

Barrera, S. D. (2000). Linguagem oral e alfabetização: Um estudo sobre variação lingüística $e$ consciência metalingüística em crianças de primeira série do ensino fundamental. Tesis Doctoral no publicada. São Paulo: Universidade de São Paulo.

Bryant, P. y Goswami, U. (1987). Beyond grapheme-phoneme correspondence. Cahiers de Psychologie Cognitive, 7, 439-443.

Bradley, L. y Bryant, P. (1991). Phonological skills before and after learning to read. En S. Brady y D. Shankweiler (Eds), Phonological Processes in
Literancy (p.p. 45-60). Hillsdale, N.J.: Elbraum.

Bryant, P. y Bradley, L. (1983). Categorizing sounds and learning to read: a causal connection. Nature, 301, 419-421.

Bryant, P. y Bradley, L. (1998). Problemas Infantiles de Lectura. Madrid: Alianza.

Bryant, P., Maclean, M. y Bradley, L., Crossland, J. (1989). Nursery rhymes, phonological skills and reading. Journal of Child Language, 16, 407-428.

Defior, S. y Tudela, P. (1994). Effect of phonological training on reading and writing acquisition. Reading \& Writting, 6, 299-320.

Cardoso-Martins, C. y Duarte, G. (1994). Preschool children's ability to disregard meaning and pay attention to the phonological properties of speech: Some discrepant findings. British Journal of Developmental Psychology, 12, 429-438.

Cardoso-Martins, C. (1995). Sensitivity to rhymes, syllables, and phonemes in literacy acquisition in Portuguese. Reading Research Quarterly, 30, 808-828.

Carrillo, M. S. y Serrano, J. M. (1992). Desarrollo Metafonológico y Adquisición de la Lectura: Un Programa de Entrenamiento. Madrid. Ministerio de Educación y Ciencia. Madrid: CIDE.

Carrillo, M. S. (1994). Development of phonological awareness and reading acquisition: A study in Spanish language. Reading \& Writing, 6, 279298.

Cook, G. (2000). Language play, language learn. Oxford: Oxford University Press.

Goswami, U. y Bryant, P.(1990). Phonological skills and learning to read. London: Erlbaum.

Goswami, U.(1995). Phonological development and reading by analogy: What is analogy, and what is not? Journal of Research in Reading. Special Issue: The contribution of psychological research, 18, 139-145.

Goswami, U. (1999). Causal connections in beginning reading: the importance of rhyme. Journal of Research in Reading, 22, 217-240.

Defior, S. (1994). La conciencia fonológica y la adquisición de la lectoescritura. Infancia $y$ Aprendizaje, 67-68, 91-113. 
Defior, S. (1996). Una clasificación de las tareas utilizadas en la evaluación de las habilidades fonológicas y algunas ideas para su mejora. Infancia y Aprendizaje, 73, 49-63.

Ferreiro, E. y Teberosky, A. (1979). Los Sistemas de Escritura en el Desarrollo del Niño. Buenos Aires: Siglo XXI.

Ferreiro, E. (1986). Proceso de alfabetización. La alfabetización en proceso. Buenos Aires: Centro Editor de América Latina.

Ferreiro, E. (1996). The acquisition of cultural objects: The case of written language. Prospect, XXVI, 131-140.

Ferreiro, E. (2000). Entre la sílaba oral y la palabra escrita. Revista Infancia y Aprendizaje, 89, 25-37.

Ferreiro, E. (2002). Escritura y oralidad: unidades, niveles de análisis y conciencia metalingüística. En E. Ferreiro (Ed.), Relaciones de (in) dependencia entre oralidad y escritura, (pp. 151-171). Barcelona: Gedisa.

Ferreiro, E. y Vernon S. (1992). La distinción de palabra/ nombre en niño de 4 y 5 años. Infancia y Aprendizaje, 58, 15-28.

Jiménez, J. (1992). Metaconocimiento fonológico: estudio descriptivo sobre una muestra de niños prelectores en edad preescolar. Infancia $y$ Aprendizaje, 57, 49-66.

Morais, J. (1991). Phonological Awareness: A bridge between language and literacy. En D. J. Sawyer y B. J. Fox (Eds), Phonological awareness in reading. The evolution of current perspective, (pp. 3171). Nueva York: Springer-Verlag.

Morais, J. (1994). El arte de leer. Madrid: Aprendizaje Visor.

Moreira, N. y Pontecorvo, C. (1998). Chapeuzinho/ Cappuccetto: variaciones gráficas y norma ortográfica. En E. Ferreiro, (Ed.) Caperucita roja aprende a escribir. Estudios psicolingüísticos comparativos en tres lenguas (pp. 89-121). Colección Lea. Barcelona: Gedisa.
Olson, D. (1994). The world on paper. The conceptual and cognitive implications of writing and reading. Cambridge: Cambridge University Press.

Rego, L. L. B. y Bryant , P. (1993). The connection between phonological, syntactic and semantic skills and children's reading and spelling. European Journal of Psychology of Education, 8, 235-246.

Ribeiro, S. y Teberosky, A. (2007). Extracción y segmentación de palabras y alfabetización inicial. En E. Diez-Itza (Ed.), Estudios de desarrollo del lenguaje y educación (pp.357-362), V Congreso Internacional de Adquisición del Lenguaje. Universidad de Oviedo: ICE.

Share, D. L. (1995). Phonological recoding and selfteaching: Sine qua non of reading acquisition. Cognition, 55, 151-218.

Snowling, M. (1980). The development of grapheme-phoneme correspondence in normal and dyslexic readers. Journal of Experimental Child Psychology, 29, 294-305.

Stanovich, K. E. (1986). Matthew effects in reading. Some consequences of individual differences in the acquisition of literacy. Reading Research Quarterly, 21, 360-406.

Tolchinsky, L., Teberosky, A. y Matas, J. (1993). Phonological knowledge and writing: A developmental study in two writing systems. En L. Verhoeven y A. Teberosky (Eds.), Proceeding of the workshop on understanding early literacy in a developmental and cross-linguistic approach (pp. 83-127), Volume II. Wassenaar, Netherlands: European Science Foundation.

Vernon, S. (1998). Escritura y conciencia fonológica en niños hispano-parlantes. Infancia $y$ Aprendizaje, 81, 105-120.

Vernon, S. y Ferreiro, E. (1999). Writing development: A neglected variable in the consideration of phonological awareness. Haward Educational Review, 69, 395-415.

Manuscrito recibido: 10/07/2010

Revisión recibida: 14/09/2010

Manuscrito aceptado: 16/09/2010 